\title{
Public perceptions of the role of dietary and other environmental factors in cancer causation or prevention
}

\author{
Katrine I Baghurst, Peter A Baghurst, Sally J Record
}

\begin{abstract}
Study objective-The aim was to establish the public's perception of the relative importance of various environmental risk factors for cancer.

Design-A postal survey was undertaken using a questionnaire to assess the public's knowledge of cancer morbidity and mortality and the role of lifestyle and genetic risk factors. Sociodemographic data were also collected.
\end{abstract}

Setting-The survey was completed in the state of South Australia.

Participants-A random sample of 1500 names were selected from the electoral rolls of the state. These rolls contain the names of all Australian citizens over the age of 18 years. A response rate of $73 \%$ was achieved.

Measurements and main results-The results of the survey showed that the knowledge base of the community was generally high, with few differences across sociodemographic groups. The relative importance of cancer as a contributor to mortality was, however, overestimated and the potential for "cure" underestimated. The role of both diet and cigarette smoking in cancer promotion was widely recognised but there was an overemphasis on the importance of pollution of the food supply compared to imbalance of nutrients. Respondents were more able to assign risk in relation to diet using a food based assessment, compared to a nutrient approach. There was wide acceptability that lifestyle change could have a profound effect on the cancer profile of the community.

Conclusions-With the relatively high degree of awareness and acceptance of lifestyle factors as cancer risk determinants, campaigns which involve skill transfer and removal of barriers to change would appear to be the most relevant approach to improvement in community behaviour.

CSIRO Division of Human Nutrition, PO Box 10041 Gouger St, Adelaide SA 5000, Australia

K I Baghurst

P A Baghurst

S J Record

Correspondence to: Dr K Baghurst

Accepted for publication March 1991
In recent years, there has been an increasing interest in, and knowledge gained about, the role that environmental factors, including diet, can play in the promotion or amelioration of the cancer process. A detailed assessment of the relative risks of these various factors was undertaken by Doll and Peto in $1981 .{ }^{1}$ In their review they estimated that some $35 \%$ of cancers in industrialised countries such as the United States, Great Britain, or Australia could be attributed to dietary factors. They also cautioned, however, that this was a best estimate figure and that the true figure could lie anywhere between 10 and $70^{\circ}{ }_{0}$. The links between dietary factors and cancer causation or prevention have since been further explored and increasing knowledge in this area has led to the development of various dietary guidelines for cancer prevention. In general, the recommendations of health authorities include increased consumption of foods rich in micronutrients and fibre such as wholegrain cereals, fruits, and vegtables (particularly the red-yellow, leafy green, and cruciferous varieties), and decreases in the usage of high fat, pickled or cured foods, and alcohol. ${ }^{2-4}$ Guidelines such as these can provide a useful framework for the development of public education and intervention initiatives. However, to maximise the effectiveness of such initiatives, it is first necessary to understand the concerns and salient beliefs of the target population. This is particularly so in the area of cancer risk where the public are subjected to a barrage of media information which is often conflicting or misleading.

However, a review of published reports shows that little research has been carried out into the public's perceptions of cancer except in areas related to the value of screening procedures, ${ }^{5-10}$ or in people who either have the disease, have a close relative with the disease, ${ }^{1112}$ or are health professionals. ${ }^{13}$ There is little or no information about the knowledge or concerns of the general public about the relative importance of various risk factors or of their perception of survival rates or trends in cancer morbidity and mortality.

To this end, a postal survey of a random sample of the adult population of the state of South Australia was carried out in 1988/1989.

\section{Methods}

A random sample of 1500 names was selected from the electoral rolls for the state of South Australia, representing a 1:500 sample of that state's adult population. As voting at state or federal elections is compulsory in Australia, the electoral rolls contain the names and addresses of all Australian citizens over the age of 18 years.

Each person selected was sent a questionnaire booklet through the post with a stamped, self addressed envelope for return of questionnaires, a covering letter explaining the purposes of the study, and the number of a toll free telephone line for enquiries. Two weeks later, a reminder post card was sent to those who had not responded and this was followed, a further two weeks later, by a replacement questionnaire and letter. 
The questions asked in the booklet fell into four categories-those related to respondents' perceptions of morbidity and mortality associated with cancer; those relating to their perception of personal risk status; those relating to the importance of specific risk factors for three major cancer sites; and those relating to their perception of the importance of a wide range of potential environmental carcinogens, particularly those related to diet. Sociodemographic data relating to age, sex, occupational status and area of residence (metropolitan or rural) were also collected and the significances of differences in response between the various subpopulations was determined using maximum likelihood $\chi^{2}$ tests.

\section{Results}

After taking into account those who had moved to another state or overseas or those who had died since the electoral roll was compiled, a $73 \%$ response rate was achieved for this survey. The age/sex/occupational status profile of the respondents mirrored closely that of the state population. Approximately half the repondents came from the state capital and half from the smaller cities, townships, and rural areas. Eight percent of the respondents had themselves had cancer and $33 \%$ had a close relative who had suffered from cancer at some stage.

\section{PERCEPTIONS OF CANCER IN AUSTRALIA}

Figure 1 shows the mean responses to questions relating to respondents' perceptions of the percentage of deaths in Australia due to cancer; whether they felt that the rate of cancer incidence was increasing; what percentage of cancers they felt were "curable", and their perceptions of the relative roles of lifestyle or genetic factors in cancer risk, both in general and at a personal level.

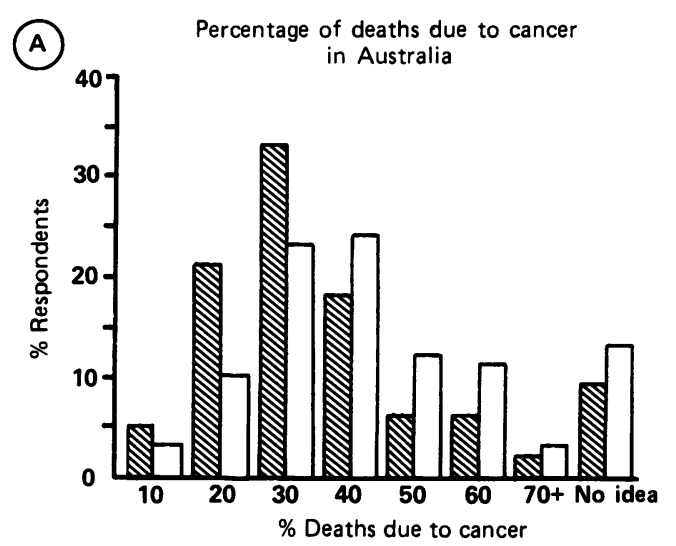

Overall, there was a suprisingly high degree of consistency in the responses across gender, age bands, occupational status, and areas of residence. Where there were sociodemographic variations, these are outlined in the text.

\section{Cancer mortality, incidence, and trends}

When asked what percentage of deaths they thought were due to cancer, $11 \%$ of respondents stated that they had "no idea" (fig 1A). The majority of respondents $(73 \%$ men; $57 \%$ women) perceived that cancer was responsible for between $20 \%$ and $30 \%$ of deaths (actual rate in South Australia $=23 \%$ ), but the response curve was strongly skewed in favour of the upper end of the scale. Women perceived cancer as a somewhat greater contributor to mortality than men, with an average (mean) response of $44 \%$ of all deaths compared to $37 \%$ for men; however this difference was not statistically significant. In two of the sociodemographic groups, namely older women $(19 \% v 8-10 \%$ in the men and younger women, $\left.\chi^{2}=8.37, p<0.04\right)$ and men of lower occupational status $(17 \% v 6-9 \%$ in other occupational groups, $\left.\chi^{2}=7.13, \mathrm{p}<0.05\right)$, there was a higher degree of uncertainty about the importance of cancer to overall mortality, with over twice the number of subjects responding "no idea" and, in those who did not give a response, a higher perception of the importance of cancer in relation to mortality. For example, $21 \%$ of the men in the lowest quintile for occupational status rated cancer as being responsible for greater than half of the deaths in Australia, while only $8 \%$ of those in the upper occupational group responded in this way $\left(\chi^{2}=5.25, p<0.02\right)$. The importance of cancer to overall mortality in Australia was generally overestimated, possibly reflecting the high level of concern in the community associated with this disease. It is also possible that the
Figure 1 Respondents' perceptions of cancer mortality, survival, and the potential effect
improved lifestyle
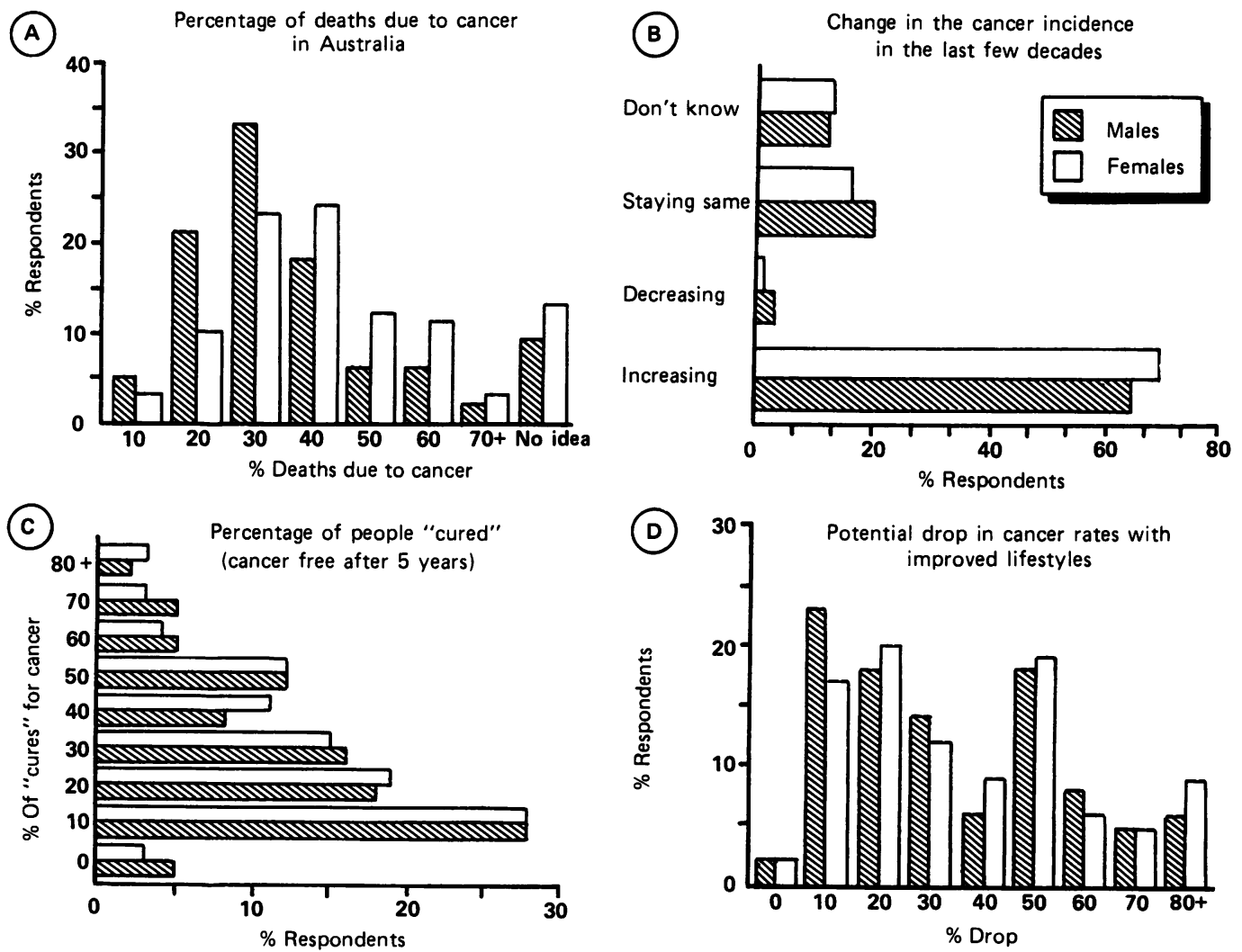
differential responses between social groupings concerning the importance of cancer to overall death rates might reflect their knowledge base or their actual experiences within their social grouping. Thus men from lower social groups, who have the highest smoking rates of all sectors of the Australian community, might have a heightened awareness of and concern about a disease such as lung cancer compared to men from upper social groups or women.

When asked about their perception of trends in cancer incidence over the past decade or so, $67 \%$ of subjects felt that the rate of cancer deaths was increasing in Australia, with $18^{\circ}{ }_{0}$ feeling it to be stable and $2 \%$ perceiving it to be declining (fig 1B). In fact, there has been an increase in cancer incidence of some $5 \%$ in men over the past decade and a $12 \%$ increased incidence in women, although the age standardised death rate has gone down slightly in men and up by only $6 \%$ in women. There were no significant sociodemographic variations in the responses to this question.

"Curability"

When asked about their perception of the "curability" of cancer, the responses were wide ranging but, again, showed no consistent age/sex/ occupational status variation. About half the respondents felt that $20^{\circ}$ or less of cancers (excluding minor skin cancers) were "curable", using five year survival free of cancer as the measure of "cure"; with a further $30 \%$ believing the "cure" rate to be between 30 and $40^{\circ}$ o (fig 1C). In fact, the figures for Australia in 1988 was substantially higher than most respondents thought, averaging just over $45^{\circ}$ o survival for men and just over $55 \%$ for women, excluding minor skin cancers.
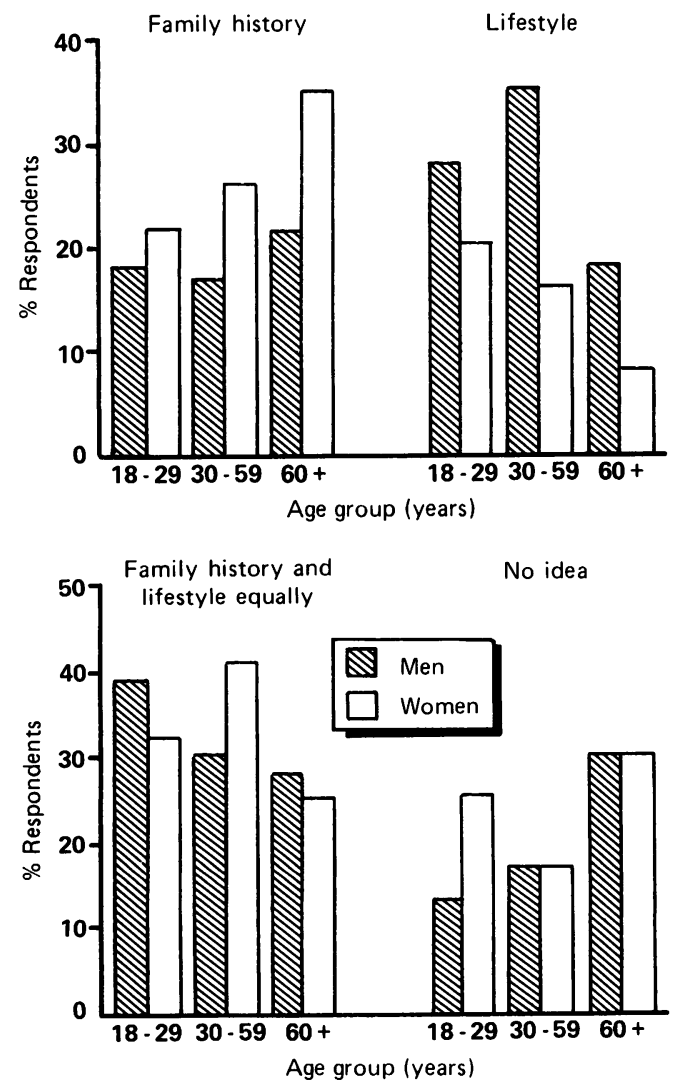

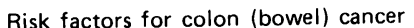

Right food Family history Stress Body chemistry Overweight Exercise

Alcohol

Cigarettes

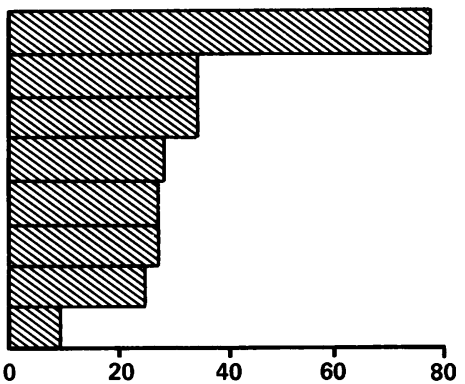

Risk factors for lung cancer

Cigarette smokin Family history

Body chemistry

Exercise

Stress

Alcohol

Overweight

Right food

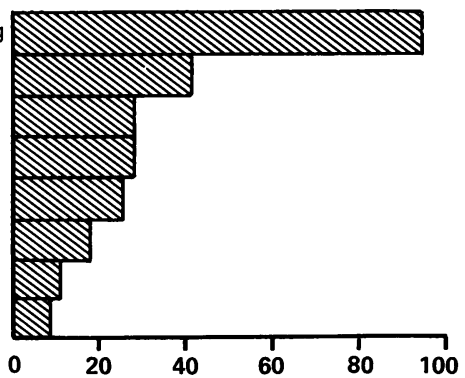

Risk factors for breast cancer

Body chemistry Family history Stress

Cigarettes

Right food

Overweight

Exercise

Alcohol

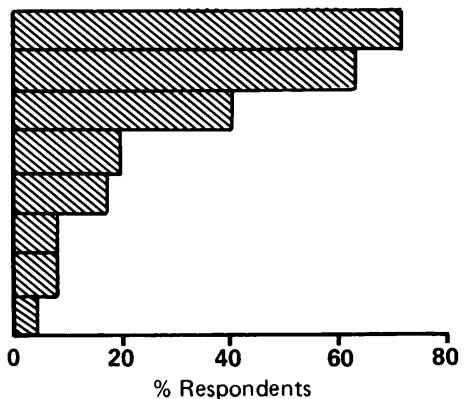

Figure 3 Respondents' perceptions of the major risk factors for specific cancer sites

Lifestyle, genetics, and personal risk

When asked what potential they thought lifestyle improvements would have to reduce the rate of cancer in Australia, about half the respondents felt that we could achieve a $10-30^{\circ}{ }_{0}$ drop in the rate, but just over one third of respondents put the potential improvement at $50 \%$ or more (fig 1D). Again, there were no marked sociodemographic variations. This result appears to show a high degree of acceptance that many cancers are avoidable by appropriate lifestyle change.

When asked about their perception of their personal risk for cancer, $45^{\circ}$ of respondents felt themselves to be at "average" risk, $28^{\circ}{ }_{0}$ below average, and $10^{\circ}$ o above average. The only sociodemographic variation was in a higher degree of uncertainty in those of lower occupational status $\left(\chi^{2}=6.36, p<0.01\right)$.

When asked whether they felt that their own risk status related more to lifestyle factors or genetic/family history factors, there were differences in the responses between sociodemographic groups with men $\left(\chi^{2}=25.93\right.$, $\mathrm{p}<0.001)$ and younger people $\left(\chi^{2}=8.24\right.$, $\mathrm{p}<0.004$ ) giving more importance to lifestyle, and women and older people to genetics/family history (see fig 2). There were no differences between occupational or residential groups. 
RELATIVE IMPORTANCE OF RISK FACTORS FOR SPECIFIC CANCER SITES

To establish whether the public discriminated between sites of cancer in relation to risk factors, participants were asked to list, in order of importance, up to three risk factors from a list of nine provided in the questionnaire (see fig 3 ) for cancer of the lung, breast, or colon (bowel). Respondents were also allowed to enter additional risk factors if they wished.

There was a differing perception of the major risk factors for cancer at the various sites, with family history rating highly in all cases, "cigarettes" being the major nominee for lung cancer, "body chemistry" for breast cancer, and "eating the right foods" for colon cancer. Stress was also rated highly as a risk factor for breast and colon cancer. Alcohol consumption was rated fairly low in each case.

RELATIVE IMPORTANCE OF ENVIRONMENTAL RISK FACTORS FOR CANCER

The major part of the study was comprised of two sets of questions which were asked to assess the

Table I Percentages of respondents who considered the listed items to be very important in relation to increased cancer risk (in order of importance according to respondents)
Table II Perceptions of the effects of various foods and nutrients on cancer risk

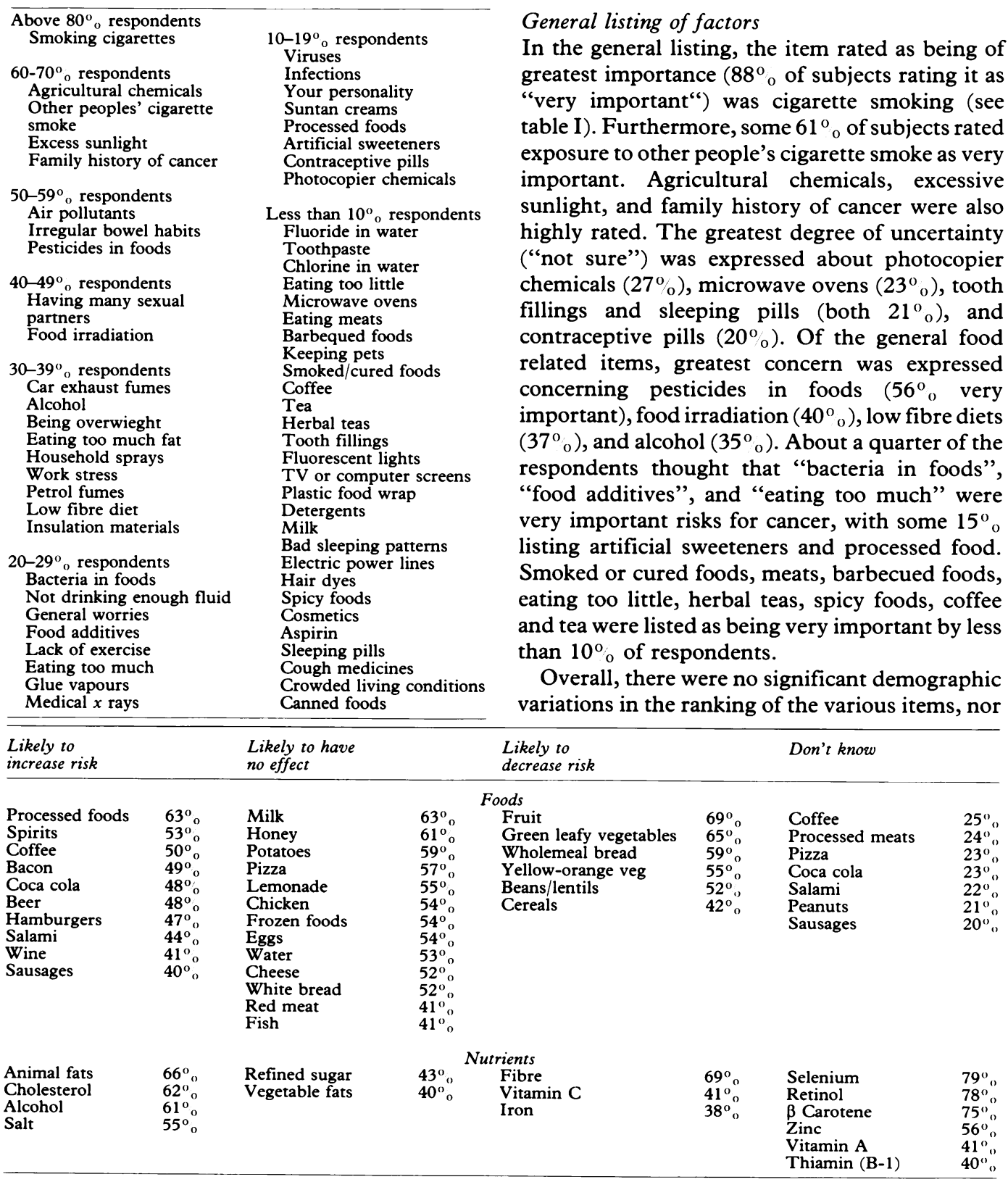

General listing of factors "very important") was cigarette smoking (see table I). Furthermore, some $61^{\circ}{ }_{0}$ of subjects rated people's cigarette smoke as very chemicals $\left(27^{\circ}\right)$, microwave ovens $\left(23^{\circ}\right)$, tooth fllings and sleeping pills (both $21^{\circ}{ }_{0}$ ), and (d) respondents thought that "bacteria in foods", " listing artificial sweeteners and processed food. Smoked or cured foods, meats, barbecued foods, $10^{\circ}$ of respondents. an $10 \%$ of respondents.

Overall, there were no significant demographic or

respondents' concerns about a wide range of potential environmental carcinogens. The first listing contained some 63 general items (see table I). These included a range of household items, environmental pollutants, pills, medications, and infections and lifestyle factors, including some generic food and drink items. Respondents were asked to score each item as "probably not very important", "quite important", "very important", or "I'm not sure", in relation to their role in the initiation or promotion of cancer. Non-preventable risk factors such as family history were also included in this list for comparison. The second listing concentrated entirely on individual foods and nutrients. In this instance, respondents were asked to indicate whether they felt that each of the 38 food/drink items or 21 nutrients "may increase", "may decrease", or "probably have no effect" on cancer risk or whether they had "no idea" (see table II). Food items were included in both lists to assess how these related, in perceived importance, to other potential environmental risk factors. 
were there any statistically significant differences between males and females, young and old, those of high or low occupational rank, or those living in metropolitan versus country areas in their overall level of concern. That is to say that the numbers of items ranked of "very", "quite", "not important", or "not sure" were similar in all demographic groups, as was the relative ranking of items.

\section{Foods and nutrients}

As shown table II, the individual foods, food types, or drinks which were most commonly perceived to increase cancer risk were processed foods, spirits, coffee, bacon, beer, coca cola, hamburgers, and sausages. Those most commonly listed as potentially decreasing cancer were green leafy vegetables, fruit, wholemeal bread, yellow-orange vegetables, beans/lentils, and cereals. Those most commonly felt to have no effect were milk, honey, potatoes, tea, lemonade, chicken eggs, water, and white bread. Most uncertainty about risk status was expressed in relation to coffee, processed meats, pizza, coca cola, salami, peanuts, and sausages.

The nutrients thought potentially to increase cancer risk were animal fats, cholesterol, alcohol, and salt. Those thought most likely to decrease the risk were fibre, vitamin C, and iron. Again, there were few consistent differences in the responses both to food related and nutrient related questions across sociodemographic groups.

In comparison to the results relating to foods, there was a very high degree of uncertainty in relation to nutrients and their role in cancer risk. For example, although less than $25 \%$ of people responded "don't know" in relation to any of the listed foods, over $70 \%$ did this for selenium, retinol, or $\beta$ carotene, just under $60 \%$ for zinc, and about $40 \%$ for vitamin $A$ and thiamin (see table II).

\section{Discussion}

In Australia, as in many other industrialised nations, coronary heart disease has been the main cause of death in the community for the past four or five decades. In recent years, however, the rate of death from coronary heart disease has dropped in many industrialised countries. ${ }^{14}$ In Australia the drop has been over $50 \%$ since the peak figures of the early $1970 \mathrm{~s}^{15}$ As a result cancer, which is already the second leading cause of death and which is gaining in relative importance as a contributor to mortality, is receiving much more attention in public health programmes as we gain a better understanding of its preventive nature.

In the past decade, age standardised cancer incidence in South Australia has risen some 12\% for women and $5 \%$ for males. ${ }^{16}$ In comparison, mortality has risen by only $6 \%$ in women while dropping by $4 \%$ in males. The increase in incidence has been variable across cancers, the most marked being for the relatively rare lip cancer (up $56 \%$ in males, $193 \%$ in women; probably due to excess exposure to sunlight), melanoma, again sunlight related (up $62 \%$ in men and $32 \%$ in women), rectal cancer (up $30 \%$ in men, $20 \%$ in women), lung cancer $(58 \%$ increase in women compared to a $17^{\circ}$ drop in menrelating to changing cigarette smoking patterns), and female breast cancer (up $7^{\circ}{ }_{0}$ ). Cervical cancer incidence over the past decade has increased in younger women by some $40^{\circ}{ }_{0}$ but there was a compensatory reduction of some $37^{\circ}$ in those over 49 years. Incidence rates for colon cancer, which is one of the most common cancers, have remained steady. Stomach cancer incidence has decreased by some $10 \%$ over the past decade, and pancreatic cancer, which has a high mortality rate, has fallen by $20 \%$ in males, a drop possibly related to reduction in smoking. In 1988, of all cancers, prostate and lung cancer had the highest incidence figures for men ( $16 \%$ each), followed by colon $(8 \%)$ and melanoma $(6 \%)$. In women, breast was the major site $(24 \%)$, followed by colon $\left(11^{\circ}{ }_{0}\right)$, melanoma $(7 \%$ ) and lung $(6 \%)$. Overall, there was a $35^{\circ}$ five year survival for men and $48^{\circ}{ }_{0}$ for women from all sites in the $1987 / 88$ period and deaths from cancer were responsible for $23 \%$ of all deaths. The majority of respondents to our survey had a good understanding of the relative importance of cancer as a contributor to deaths, most respondents perceiving it to be responsible for between $20 \%$ and $30 \%$ of deaths. However, those who did not nominate in this range, and in particular young men from lower occupational groups, tended to overemphasise its importance. Over two thirds of the respondents also recognised that cancer rates were continuing to increase. "Curability" of cancer was underestimated, with half the respondents thinking that less than $20 \%$ of cancers were "curable" using the five year survival criterion. Belief in the ability to reduce cancer incidence through lifestyle change in the community was, however, encouragingly strong across all sectors of the community. However, younger people were more inclined to believe that their own risk status related to lifestyle than older respondents who had a stronger belief in the role of genetics. Interestingly, there were no social status differences in belief about the relative role of lifestyle and genetics in personal risk, implying that the differences that do exist in risk behaviour between social groups in Australia, such as occur with diet ${ }^{17}$ or smoking, ${ }^{18}$ probably relate more to specific skills and/or the existence of barriers to change than to lack of generic belief in the power of lifestyle change as a tool in cancer prevention.

Questions relating to risk factors for specified cancers also showed that the public did have a concept of the variation that occurs in site specific cancer risk behaviours. For example, acceptance of the role of lifestyle factors such as diet in colon cancer, or cigarette smoking in lung cancer, was high, but not specially so for breast cancer where these and other lifestyle factors were rated secondary to "body chemistry" and family history. In general, cigarette smoking, including passive smoking, was seen as the dominant risk factor for cancer, with agricultural chemicals, sunlight, and family history completing the top five in the general listing of risk factors.

In many ways, the ranking given by the public to the various environmental risk factors did reflect the scientific assessment of Doll and Peto. ${ }^{1}$ In their review, the major avoidable environmental factor was assessed to be diet, with 
a best estimate of $35^{\circ}$ or for proportion of deaths attributable. Second came cigarettes at $30 \%$ attributable deaths, followed by infection $\left(10^{\circ}{ }_{\mathrm{o}}\right)$; reproductive and sexual behaviour $(7 \%)$; occupational exposure $\left(4^{\circ}{ }_{0}\right)$; geophysical factors - including sunlight $\left(3^{\circ}{ }_{0}\right)$; alcohol $\left(3^{\circ}{ }_{0}\right)$, pollution $(2 \%)$; medicines and medical procedures $\left(1^{\circ}{ }_{0}\right)$; and food additives and industrial products (both less than $1 \%$ ). Compared to this scientific assessment, respondents attached less importance to the role of diet relative to cigarettes, and more importance than Doll and Peto to agricultural chemicals, air pollutants, and pesticides in foods, the occurrence of which, according to Doll and Peto "seems unimportant". It should be remembered, however, that Doll and Peto's review was carried out in the early 1980s when there was little scientifically documented evidence of the role of these types of environmental pollutants, possibly because of the technical difficulties of studying this area. Evidence of the role of agricultural chemicals, air pollutants, and pesticides in cancer causation is still limited but concerns about these factors cannot be dismissed as yet. The role of reproductive behaviour was recognised by the public, as well as that of sunlight, which, in Australia, has much more relevance to cancer risk than in many of the industrialised countries of the northern hemisphere. The roles of infections and viruses were not rated highly in the survey although Doll and Peto gave them both a relatively high attributable risk ( $5 \%$ for each). They did, however state in their review that their estimates for these two risk factors were particularly approximate- being based on limited evidence.

Of all the food related items, pesticide contamination and food irradiation were the most highly rated risks, but food additives, which have received a great deal of media coverage in Australia, were not seen to be one of the major risks in relation to cancer. It was interesting to note the relatively low concern about food additives in relation to cancer, since this item has been ranked as highly important among the general dietary concerns of South Australians in previous surveys. ${ }^{19}$ It is also interesting to note that items such as coffee and artificial sweeteners, which have received a great deal of media cover over the past decade or so in relation to cancer, were rated fairly low as potential cancer risks, in line with Doll and Peto's assessment. However when specified in the food listings, coffee was listed by a substantial proportion of respondents as a potential dietary risk for cancer.

Overall, the respondents appeared to show a good awareness of the various patterns of food intake that might influence cancer risk, although they appeared to have limited awareness of the potential problem of cured, salted, or pickled foods and they were overly concerned with issues such as food irradiation and pesticides in foods compared to their concern with overall nutrient balance. Although current pesticide levels in foods are not considered by the "experts" to confer a significant risk for cancer, they still remain of high concern to the general public and may therefore have to be addressed in education programmes concerning the role of diet in cancer prevention.
It is difficult to assess how much of the apparent awareness of diet relates to specific knowledge about dietary cancer risk and how much merely reflects general concepts about certain types of foods being "good for you" or, conversely, "bad for you"-be it in the context of cancer, heart disease, diabetes, hypertension, osteoporosis, or any one of the major chronic disease entitities. It is possible, for instance, that the negative responses to cholesterol and salt risk may reflect the status of these nutrients in relation to cardiovscular disease and hypertension rather than cancer per se. There is some evidence, summarised in the recent report of the National Research Council of the United States, ${ }^{20}$ that both cholesterol and salt may increase cancer risk, but the evidence is limited and is unlikely to have reached the public domain. Conversely, the positive, but erroneous, role seen for iron in cancer prevention or amelioration probably reflects its high status in relation to blood formation and prevention of anaemia, rather than cancer. At the same time it was of interest to note that refined sugars, which have received widespread negative media coverage in the past decade or so, were not perceived as increasing cancer risk, showing some degree of disease specific discrimination in the public judgement of nutrients.

In general, respondents were less certain of the role of nutrients, as opposed to foods, in cancer causation. This seemed to reflect a lack of familiarity with the names of various nutrients, as respondents were more sure in their responses to more widely discussed nutrients such as fat and fibre. These findings suggest that health promotion programmes aimed at reducing diet related cancer risk are more likely to be effective if they build on the current food based orientation of the community. It is fortuitous that the pattern of food consumption that needs to be encouraged to prevent or ameliorate cancer is congruent with the well established and discussed pattern for reduction of coronary risk, with the additional emphasis on fruits and vegetables rich in vitamins $A$ and $C$.

Surveys of the dietary intakes of random samples of the Australian ${ }^{21}$ and United States 22 populations have shown that although women, older age groups, and people of higher social status do have diets more in line with guidelines for reduction in cancer risk, the current intake of most people, regardless of age, sex or occupational status, deviates widely from recommendations and that intervention is needed in all sectors in the community. This survey has shown that all sectors of the public are receptive to the concept of risk reduction through lifestyle and dietary change and that in the dietary arena, they are more likely to understand and thus respond to a food based approach. To implement behavioural change in the community, it will be necessary to build on the current knowledge base to address the societal and personal barriers to change, such as costs and availability of recommended foods or, for other lifestyles factors such as sunlight exposure, of items such as suntan creams and protective clothing. Skills development in areas such as how to prepare nutritious and time saving low cost meals or how to overcome addictive behaviours such as smoking, will also be 
necessary. From our results, these latter issues, rather than differences in beliefs about the intrinsic value of various dietary or lifestyle choices relating to cancer risk, appear to be the more likely determinants of sociodemographic variations in community behaviour patterns, as, with few exceptions, all sectors of the adult community appeared equally aware of the avoidable risk factors for cancer.

1 Doll R, Peto R. The causes of cancer: quantitative estimates of avoidable risk of cancer in the United States today. 1 Natl Cancer Inst 1981; 66: 1193-265.

2 National Research Council. Diet, nutrition and cancer. Washington DC: National Academy Press, 1982.

3 American Cancer Society. Nutrition, common sense and cancer. New York: American Cancer Society, 1985.

cancer. New York: American Cancer Society, 1985.
American Institute for Cancer Research. Planning meals that lower cancer risk-a reference guide. Washington DC: that lower cancer risk-a reference guide. Washingt

5 Champion VL. Attitudinal variables related to intention, frequency and proficiency of breast self-examination in women 35 and over. Res Nurs Health 1988; 11: 283-91.

6 Celentano DD, Klassen AS, Wesmann CS, Rosenshein NB. Cervical cancer screening practices among older women; results from the Maryland Cervical Cancer CaseControl Study. F Clin Epidemiol 1988; 41: 531-41.

7 Reno DR. Men's knowledge and health beliefs about testicular cancer and testicular self-examination. Cancer Nurs 1988; 11: 112-7.

8 Rutledge DN Davis GT. Breast self-examination compliance and the health-belief model. Oncol Nurs Forum 1988; 15: 175-9.

9 Walker LR Glanz K. Psychosocial determinants of breast self-examination. Am $\mathcal{F}$ Prev Med 1986; 2: 169-78.
10 Champion VL. The relationship of breast self-examination to health belief model variables. Res Nurs Health 1987; 10 375-82.

11 Sack H, Bischof J. The knowledge of cancer patients about their disease. Strahlenther Onkol 1988; 164: 581-92.

12 MacKillop WJ, Stewart WE, Ginsburg AD, Stewart SS Cancer patients' perceptions of their disease and its treatment. $\mathrm{Br} \mathcal{F}$ Cancer 1988; 58: 355-8.

13 American Cancer Society. 1989 Survey of physicians' attitudes and practices in early cancer detection. $C A 1990$ 40: 77-101.

14 Havlik RJ Feinleib M. Proc Conf on decline in Coronary Heart Disease Mortality. US Dept Health, Education and Welfare Public Health Service. NIH Publication No 791610. Washington: National Institutes of Health, 1979.

15 Leeder SR, Gibberd R, Dobson AJ, Lloyd DM. Declining mortality rates from ischaemic heart disease in Australia. Aust NZ F Med 1984; 14: 383-94.

16 Bonett A, Roder D, Esterman A. Epidemiology of cancer in South Australia. Incidence, mortality and survival 19771988. Adelaide: South Australian Cancer Registry, 1990.

17 Baghurst KI, Crawford DC, Record SJ, Worsley A, Baghurst PA, Syrette J. The Victorian Nutrition Survey. Part 2, Nutrient intakes by age, sex, area of residence and Part 2, Nutrient intakes by age, sex, area of

18 Dobson AJ, Gibberd, RW, Leeder SR, O'Connell DL Occupational differences in ischaemic heart disease mortality and risk factors in Australia. Am $\mathcal{f}$ Epidemio 1985, 122. 283-90.

19 Crawford DA, Baghurst KI. Diet and health-a national survey of beliefs, behaviours and barriers to change in the community. Aust $\mathcal{F}$ Nutr Diet 1990; 47: 97-104.

20 National Research Council Diet and Health. Implications for reducing chronic disease risk. Washington DC: National Academy Press, 1989.

21 Baghurst KI, Record SJ, Baghurst PA, Crawford DA, Syrette JA, Worsely A. Sociodemographic determinants in Australia of the intake of food and nutrients implicated in cancer aetiology. Med f Aust 1990; 153: 444-52.

22 Patterson BH, Block G. Food choices and the cancer guidelines. Am $\mathcal{f}$ Public Health 1988; 78: 282-6. 